\title{
Energy-Optimum Throughput and Carrier Sensing Rate in CSMA-Based Wireless Networks
}

\author{
Mehmet Koseoglu and Ezhan Karasan, Member, IEEE
}

\begin{abstract}
We propose a model for the energy consumption of a node as a function of its throughput in a wireless CSMA network. We first model a single-hop network, and then a multi-hop network. We show that operating the CSMA network at a high throughput is energy inefficient since unsuccessful carrier sensing attempts increase the energy consumption per transmitted bit. Operating the network at a low throughput also causes energy inefficiency because of increased sleeping duration. Achieving a balance between these two opposite operating regimes, we derive the energy-optimum carrier-sensing rate and the energy-optimum throughput which maximize the number of transmitted bits for a given energy budget. For the single-hop case, we show that the energy-optimum total throughput increases as the number of nodes sharing the channel increases. For the multi-hop case, we show that energy-optimum throughput decreases as the degree of the conflict graph corresponding to the network increases. For both cases, the energy-optimum throughput reduces as the power required for carrier-sensing increases. The energy-optimum throughput is also shown to be substantially lower than the maximum throughput and the gap increases as the degree of the conflict graph increases for multi-hop networks.
\end{abstract}

Index Terms-Carrier sense multiple access, energy efficiency, analytical models, performance analysis, throughput

\section{INTRODUCTION}

$\mathrm{T}$ O improve the battery lifetimes of wireless devices and due to environmental considerations, the energy efficiency of wireless communication protocols has to be improved. There are many wireless communications protocols that employ a variant of the carrier sense multiple access protocol (CSMA) due to its simple and distributed nature (e.g., the IEEE 802.11 for WLANs, IEEE 802.15.4 for WPANs and B-MAC for sensor networks [1]). We here find the optimum carrier-sensing rate and throughput which maximizes the number of transmitted bits in a wireless CSMA network for a fixed energy budget.

Recently, carrier-sensing rate adaptation algorithms have been devised to achieve throughput-optimality in a CSMA network [2]. In these algorithms, each node senses the channel at a rate which increases with its packet queue length (or virtual queue length). As packet queues grow, the nodes may sense the channel at arbitrarily high rates. However, the increased energy consumption due to such increased carrier-sensing rate has not been investigated to the best of our knowledge. We here aim to quantify the relationship between sensing rate, throughput and energy consumption in a CSMA network.

We consider a saturated CSMA network where all nodes always have a packet to send and employ non-persistent

- The authors are with the Department of Electrical and Electronics Engineering, Bilkent University, Ankara TR-06800, Turkey.

E-mail:mkoseoglu@gmail.com; ezhan@ee.bilkent.edu.tr.

Manuscript received 6 May 2013; revised 21 Aug. 2013; accepted 19 Sep. 2013. Date of publication 29 Sep. 2013; date of current version 29 May 2014

For information on obtaining reprints of this article, please send e-mail to: reprints@ieee.org, and reference the Digital Object Identifier below.

Digital Object Identifier 10.1109/TMC.2013.124
CSMA [3]: If the channel is busy when a node senses the channel, it waits for an exponentially distributed duration with mean $\lambda^{-1}$ and attempt to transmit again. During the waiting time between transmission attempts, the node can be either in the idle listening state or in the sleeping state. For the rest of the paper, we will refer to the waiting time between transmission attempts as sleeping since the sleeping state is the most energy saving state. However, the proposed analysis is still applicable even when nodes perform idle listening between transmission attempts.

We are interested in the following question: What is the optimum value of $\lambda$ which maximizes the number of transmitted bits for the lifetime of the node which is limited by its energy budget. If $\lambda$ is selected too small, the nodes will rarely transmit a packet and spend most of their lifetimes in the sleep mode. In this case, a node consumes its energy budget mostly in the sleeping state albeit sleeping has minor energy consumption. A very low $\lambda$ can improve the duration of service but it will not improve the number of bits that it can transmit during its lifetime.

If $\lambda$ is selected too large, the nodes will frequently wakeup and sense the channel to transmit a packet. Although it is usually omitted in the literature, each time a node senses the channel and finds it busy, a small amount of energy is spent without making a transmission. So, a very high $\lambda$ will also result in energy inefficiency.

We find the energy-optimum carrier-sensing rate, $\lambda^{*}$, which minimizes the energy consumption per transmitted bit. The energy-optimum rate exploits the trade-off between the energy consumed for sleeping and energy consumed for carrier sensing. The energy-optimum rate leads to an energy-optimum throughput, $\sigma^{*}$, which gives the 
energy-optimum operating load for the network. To maximize the number of transmitted bits for a given energy budget, the network has to operate at a throughput of $\sigma^{*}$.

We first provide an analytical model for the energy consumption of a single-hop CSMA network, and then extend the analysis to a multi-hop network with a random regular conflict graph. For both scenarios, we analyze the energy consumed in various states such as sleeping and carriersensing. We derive the energy-optimum carrier sensing rate and the corresponding energy-optimum throughput which minimize the energy consumption per transmitted bit. The energy-optimum throughput exploits a balance between the energy consumed in the states of sleeping and carrier sensing per transmitted bit.

For the single-hop network, we show that the energyoptimum throughput is higher for larger networks because sleeping costs increase dramatically at a low throughput with the number of nodes. Also, the energy-optimum throughput increases as the power required for carriersensing reduces in proportion to the power required for sleeping. As sensing becomes less expensive, the nodes should attempt to transmit packets more frequently to minimize the energy consumed per bit.

For the multi-hop case, we show that the energyoptimum throughput depends on the degree of the conflict of graph of the network and on the power consumption of carrier sensing. We find that the energy-optimum throughput reduces as the degree of the conflict graph increases, i.e., as the interference increases. Similar to the single-hop case, the energy-optimum carrier sensing rate and the energyoptimum throughput increase as the power required for carrier sensing reduces.

In the next section, we present a review of the relevant literature. The energy consumption analyses for single-hop and multi-hop networks are given in Sections 3 and 4, respectively. We derived bounds for the energy-optimum throughput and maximum throughput for the multi-hop case in Section 5. The numerical evaluation of the proposed analysis is given in Section 6. Section 7 presents the conclusions and discussion.

\section{Related Work}

The energy efficiency of the CSMA protocol is analyzed in the context of several different standards. To evaluate the energy consumption of the IEEE 802.11 protocol, Bononi et al. [4] and Bruno et al. [5] analyzed the slotted p-persistent CSMA to evaluate the tradeoff between the throughput and the energy efficiency. A more detailed model for energy consumption for 802.11 is presented in [6]. Energy efficiency of the 802.11 protocol in a multihop setting is analyzed in [7].

Most of the MAC protocols for power-constrained devices employ the non-persistent CSMA instead of the p-persistent CSMA to eliminate idle listening. Chan et al. [8] compared the energy efficiency of the slotted non-persistent CSMA against the analysis of the p-persistent CSMA given by [4], [5]. For the IEEE 802.15.4 standard, energy consumption of the slotted non-persistent CSMA is also analyzed in [9], [10]. Similarly, energy consumption of slotted CSMA/CA is analyzed for uplink traffic in IEEE 802.15.4 networks [11].
Most of these studies assume a time-slotted version of CSMA since they are targeted for standards with slotted operation. However, we here study a continuous-time CSMA network. Also, these studies are mostly confined to a singlehop network but we perform an energy consumption analysis for multi-hop topologies as well as a single-hop network.

There is also a large body of literature on energy efficient MAC protocols for sensor networks [12]. These protocols usually focus on efficient duty cycling schemes to synchronize senders with receivers to minimize idle listening [1], [13]-[15]. In this study, we assume a perfect duty-cycling scheme in the multi-hop scenario. Such a scheme can be approximated by a secondary low power radio or by using a predictive wake-up schedule such as the recently proposed PW-MAC [16]. The results of our study provide insights about the optimum operating load of such networks.

Capacity scaling laws of multi-hop wireless networks have been investigated in [17]-[19] and throughput of CSMA networks have been investigated in [20]-[25].

Our work is also closely related to a recent line of study on the optimal-CSMA which is developed on the idealized CSMA model that we study in this paper. It has recently been shown that throughput-optimality can be achieved by a CSMA rate adaptation algorithm [26]-[28]. In these algorithms, nodes sense the channel at a rate which is a function of their packet queues (or virtual queues). As the queues of nodes grow at high loads, nodes sense the channel very frequently. Most of these carrier sensing attempts, however, will show that the channel is busy at high loads. Although a sensing attempt consumes a small amount of energy in comparison to reception of a packet, energy consumed for sensing may become a significant fraction of the total energy as the number of sensing attempts per packet increases. To the best of our knowledge, the impact of frequent carriersensing on the energy consumption has not been considered in the optimal-CSMA research and our work provides insights on the energy consumption of such algorithms.

In this paper, we propose a protocol-independent energy-consumption analysis of the non-persistent CSMA protocol for both single-hop and multi-hop networks. Our results provide closed from expressions describing the change of the energy-optimum operating point of CSMA networks as a function of the number of nodes (for single-hop networks) and network degree (for multi-hop networks). Besides, we investigate the change in the energyoptimum operating point as the ratio of powers required for carrier-sensing and sleeping changes. The parameters used in the analytical models for single and multi-hop networks presented in Sections 3 and 4 are listed in Table 1.

\section{Single-Hop Network}

We first consider a single-hop network scenario where the nodes transmit to a central base station. A timeline of the transmissions of a node in such a single-hop network can be seen in Fig. 1. The probability distributions of durations are also shown in the timeline. In the figure, node 2 transmits its second packet after two unsuccessful carrier sensing attempts. In this section, we analyze the energy consumption of such a network and obtain the energy-optimum throughput and carrier-sensing rate. 
TABLE 1

List of Notations

\begin{tabular}{c||l}
\hline Symbol & Definition \\
\hline \hline$\sigma$ & Throughput per node \\
$\sigma_{t o t}$ & Total throughput in the network \\
$\sigma^{*}$ & Energy-optimum throughput per node \\
$\sigma_{t o t}^{*}$ & Energy-optimum total throughput in the network \\
$\sigma^{\text {max }}$ & Maximum throughput per node \\
$\lambda$ & Carrier-sensing rate \\
$\hat{\lambda}$ & Normalized carrier-sensing rate \\
$\lambda^{*}$ & Energy-optimum carrier-sensing rate \\
$t_{l}$ & Packet duration \\
$t_{c}$ & Carrier-sensing duration \\
$N$ & Number of nodes \\
$P_{t}$ & Transmit power \\
$P_{r}$ & Receive power \\
$P_{c}$ & Power spent during carrier-sensing \\
$P_{s}$ & Power spent during sleeping \\
$T_{i}$ & Inter-transmission duration \\
$T_{t}$ & Time spent for transmission per packet \\
$T_{r}$ & Time spent for reception per packet \\
$T_{c}$ & Time spent for carrier sensing per packet \\
$T_{s}$ & Time spent for sleeping per packet \\
$R$ & Data transmission rate \\
$E_{p}$ & Energy consumed per transmitted packet \\
$E_{b}$ & Energy consumed per transmitted bit \\
\hline &
\end{tabular}

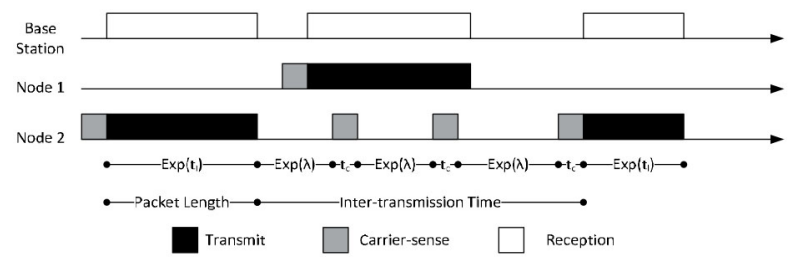

Fig. 1. Sample timeline of two nodes in a single-hop scenario.

\subsection{System Model}

In the analysis of the single-hop CSMA, we use the Markov chain model of CSMA which is proposed in [29]. This model has been frequently used in the study of optimal CSMA recently [2], [26], [27]. Based on this model, the Markov chain for a single hop scenario can be constructed as in Fig. 2 for a mean packet duration of $t_{l}$. For example, in the figure, the state $(0,0,0, \ldots, 0)$ corresponds to the state where none of the nodes are transmitting and state $(0,1,0, \ldots, 0)$ corresponds to the case where only the second node is transmitting. This model assumes instantaneous carrier-sensing, so the collisions are avoided.

Instantaneous sensing assumption allows arbitrarily large sensing rates to be handled by this model. However, in reality, carrier-sensing takes a non-negligible time which prevents the nodes to access the channel at high rates. To incorporate the sensing duration into the carrier sensing frequency while preserving the zero-collision assumption, we obtain a normalized sensing rate, $\hat{\lambda}$, by adding the sensing duration, $t_{c}$, to the mean of the carrier sensing period, $1 / \lambda$ :

$$
\hat{\lambda}=\frac{1}{\frac{1}{\lambda}+t_{c}}
$$

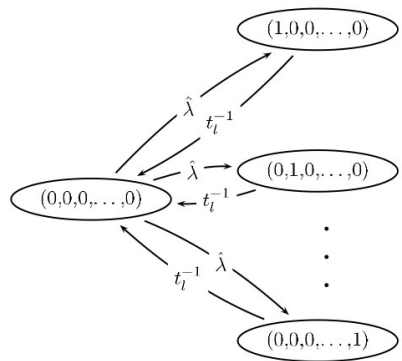

(a)

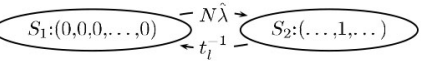

(b)

Fig. 2. (a) Markov chain for the single-hop case. The stationary probabilities of the states except the initial state gives the throughput of each node. (b) Markov chain can be condensed into two states by combining all states that correspond to active transmissions.

This implies that the carrier-sensing duration is also assumed to be exponentially distributed. Although the sensing duration is deterministic in reality, this assumption does not lead to an inaccuracy in the analysis as will be shown in Section 6 . So, as $\lambda$ approaches to infinity, $\hat{\lambda}$ approaches to $t_{c}^{-1}$ which means that the maximum sensing frequency is limited by the sensing duration.

We define the throughput of a node, $\sigma$, as the ratio of the time spent to transmit a packet to the total time. So, the throughput of nodes 1 to $N$ corresponds to the stationary probability of states $(1,0,0, \ldots, 0)$ to $(0,0,0, \ldots, 1)$ in Fig. 2(a). This Markov chain can be condensed into two states, $S_{1}$ and $S_{2}$, by combining all states that correspond to active transmissions as shown in Fig. 2(b). The stationary probability of the combined state corresponds to the total throughput of the network and can be written as:

$$
\pi_{2}=\sigma_{t o t}=\frac{\hat{\lambda} N}{\frac{1}{t_{l}}+\hat{\lambda} N} .
$$

Then, the throughput of a node in terms of $\hat{\lambda}$ and $\lambda$ is given by

$$
\sigma=\frac{\hat{\lambda}}{\frac{1}{t_{l}}+\hat{\lambda} N}=\frac{\lambda}{\frac{1}{t_{l}}+\lambda\left(N+\frac{t_{c}}{t_{l}}\right)} .
$$

The maximum throughput per node can be obtained as $\lambda$ approaches to infinity:

$$
\sigma_{\max }=\lim _{\lambda \rightarrow \infty} \sigma=\frac{1}{N+\frac{t_{c}}{t_{l}}} .
$$

The maximum throughput of a node is dependent on the number of nodes sharing the channel and the ratio of sensing duration to the packet duration.

The inverse relationship between the throughput and the carrier-sensing frequency can be obtained by taking the inverse function of (3):

$$
\lambda=\frac{\sigma}{t_{l}(1-N \sigma)-t_{c} \sigma}
$$

for $\sigma \leq \sigma_{\max }$. 


\subsection{Energy Consumption Model}

We are interested in determining the energy spent for transmission, sleeping and carrier sensing per transmitted bit. The duration between the transmissions of two successive packets consists of time spent for carrier sensing and time spent while sleeping. Since throughput equals to the ratio of the average packet duration to the sum of the average packet duration with the mean inter-transmission duration, it is possible to obtain the mean inter-transmission duration in terms of throughput by solving

$$
\frac{t_{l}}{t_{l}+E\left[T_{i}\right]}=\sigma
$$

which gives the solution as

$$
E\left[T_{i}\right]=\frac{t_{l}(1-\sigma)}{\sigma} .
$$

The inter-transmission duration includes several carriersensing periods which consists of a sleeping period and a carrier-sensing operation. If the carrier-sensing operation is unsuccessful, the sensing period is repeated. Since the mean of sleeping duration between carrier sensing attempts is $\frac{1}{\lambda}$ and the mean carrier sensing duration is $t_{c}$, it is possible to compute the share of sleeping and carrier sensing in the inter-transmission duration. The mean time spent for carrier sensing per packet can be found using (5) as

$$
E\left[T_{c}\right]=\frac{t_{l}(1-\sigma)}{\sigma} \frac{t_{c}}{\frac{1}{\lambda}+t_{c}}=\frac{t_{c}(1-\sigma)}{1-N \sigma}
$$

and mean time spent for sleeping per packet is given by

$$
E\left[T_{s}\right]=\frac{t_{l}(1-\sigma)}{\sigma} \frac{\frac{1}{\lambda}}{\frac{1}{\lambda}+t_{c}}=\frac{(1-\sigma)\left(t_{l}(1-N \sigma)-t_{c} \sigma\right)}{\sigma(1-N \sigma)} .
$$

Since the mean packet duration is $t_{l}$, i.e., $E\left[T_{t}\right]=t_{l}$, total energy consumption per packet is given by

$$
\begin{aligned}
E\left[E_{p}\right]= & \frac{t_{c}(1-\sigma)}{1-N \sigma} P_{c} \\
& +\frac{(1-\sigma)\left(t_{l}(1-N \sigma)-t_{c} \sigma\right)}{\sigma(1-N \sigma)} P_{s}+t_{l} P_{t},
\end{aligned}
$$

where $P_{c}, P_{s}$ and $P_{t}$ correspond to the power consumed while carrier sensing, sleeping and transmission, respectively. Then, energy per transmitted bit is given by

$$
E\left[E_{b}\right]=\frac{E\left[E_{p}\right]}{t_{l} R},
$$

where $R$ is the data transmission rate. Energy per bit has a single minimum for $\sigma \leq \sigma_{\max }$, so the energy minimizing $\sigma$ can be found by solving $\frac{\partial E\left[E_{b}\right]}{\partial \sigma}=0$ as

$$
\sigma^{*}=\frac{1}{\sqrt{\frac{P_{c}-P_{s}}{P_{s}} \frac{t_{c}}{t_{l}}(N-1)}+N}
$$

and the corresponding energy-optimum carrier-sensing rate can be found by substituting (12) into (3) as

$$
\lambda^{*}=\frac{1}{\sqrt{\frac{P_{c}-P_{s}}{P_{s}} t_{c} t_{l}(N-1)}-t_{c}}
$$

for $\sigma^{*} \leq \sigma^{\max }$.

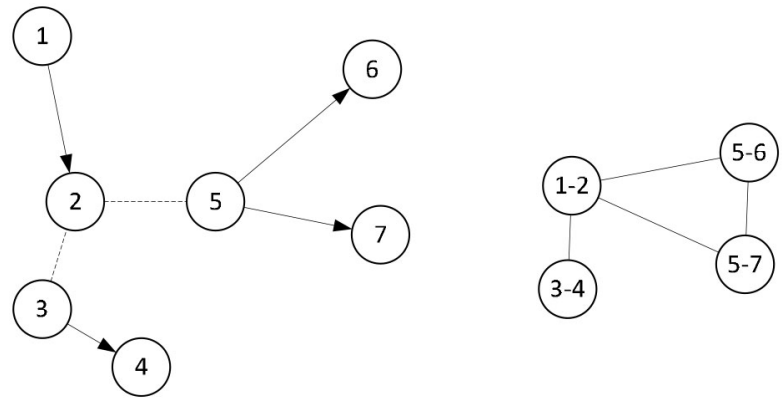

Fig. 3. Wireless network topology and the conflict graph of its links. Lines with arrows indicate the links in the network topology and dashed lines indicate that two nodes are within the interference range of each other without having a link between them.

Then, the total energy-optimum network throughput is given by

$$
\sigma_{t o t}^{*}=N \sigma^{*}=\frac{N}{\sqrt{\frac{P_{c}-P_{s}}{P_{s}} t_{c} t_{l}(N-1)}-t_{c}} .
$$

The total energy-optimum throughput decreases as $P_{c}$ gets larger in comparison to $P_{S}$ which means that $\sigma_{t o t}^{*}$ reduces as the carrier sensing gets more expensive. Also, as $N$ increases, $\sigma_{\text {tot }}^{*}$ increases because the sleeping costs increase faster than the carrier sensing costs as $N$ increases. In the limit as $N \rightarrow \infty, \sigma_{t o t}^{*} \rightarrow 1$. A detailed discussion of the properties of $\sigma_{\text {tot }}^{*}$ is presented in Section 6.1.

\section{Multi-Hop Network}

We now study a multi-hop network where nodes both transmit and receive packets unlike the single hop scenario where the nodes only transmit to a base station. Similar to the single-hop case, each node always has a packet to send and wakes up after exponentially distributed periods with mean $\lambda^{-1}$ and senses the channel. If the channel is idle, the node transmits the packet to one of its neighbors. If a node is not transmitting or receiving a packet, it sleeps to conserve energy. In our model, we assume that the sender and receiver of a packet are perfectly synchronized, both wakeup at the same time to complete the transmission. If the channel is busy when the sender wakes up, it sleeps again and wake-up after an exponentially distributed period with mean $\lambda^{-1}$. We are interested in the energy-optimum value of $\lambda$ which minimizes the energy consumption per transmitted bit, hence maximizes the number of bits that a node can transmit during its lifetime.

\subsection{System Model}

We perform our analysis on the conflict graph of links in the network. A conflict graph represents the interference relationships among links between wireless nodes in the network as shown in Fig. 3. A directed link in the network is represented by a vertex in the conflict graph and there is an edge between vertices in the conflict graph if the corresponding links are interfering with each other. In such a model, there are no hidden terminals and the propagation delays between nodes are negligible, so collisions are avoided. This model has recently been used in the design of throughput-optimal CSMA [26], [27]. 


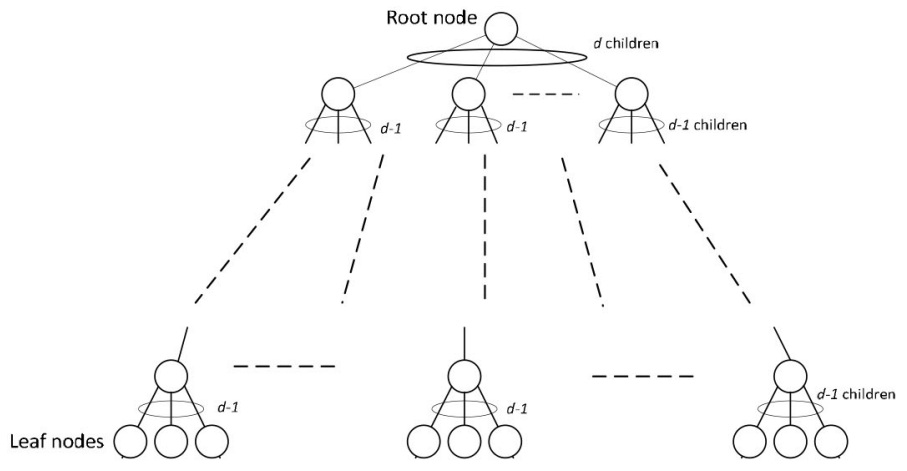

(a)

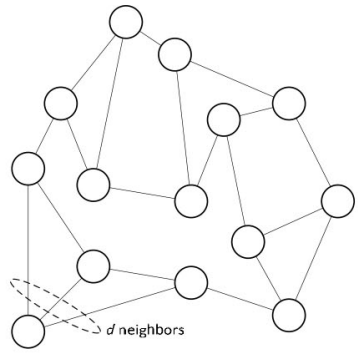

(b)

Fig. 4. (a) Cayley tree where each node has $d$ neighbors except leaf nodes. (b) Random regular graph with a degree of $d$.

For the sake of analysis, we consider a random regular conflict graph, i.e., each vertex in the conflict graph has the same number of neighbors, $d$. We assume that the transmission and reception links of a node in the wireless network correspond to a neighboring node pair in the contention graph. The nodes have saturated traffic and each node senses the channel at independent and exponentially distributed intervals with rate $\lambda$. If a node senses that there are no conflicting transmissions, it starts a transmission for an exponentially distributed duration with mean $t_{l}$.

\subsection{Energy Consumption Model}

In order to quantify the energy consumption per bit, we first have to obtain a relationship between the carriersensing rate and throughput. For the single-hop case, the throughputs can be easily obtained by solving the Markov chain given in Fig. 2. Although a similar Markov chain can be constructed for a multi-hop network, it requires enumeration of independent sets of the conflict graph which is computationally difficult. Besides, a different Markov chain has to be constructed for each topology. For that reason, we here focus on random regular conflict graphs which have a surprisingly similar throughput-sensing rate relationship with a special type of graphs known as the Cayley tree. In a Cayley tree, each node except the leaf nodes has the same number of neighbors, $d$. The relationship between throughput and carrier sensing rate in a Cayley tree graph is investigated in the context of loss networks by Kelly [30]. The random regular and Cayley tree conflict graphs can be seen in Fig. 4.

In this analysis, all non-leaf nodes have the same channel sensing rate whereas the channel sensing rates of leaf nodes are adjusted so that they have the same throughput with internal nodes. The relationship between the throughputs of nodes, $\sigma$, and the channel sensing rate of internal nodes, $\lambda$, is obtained using a fixed point equation. We here only present the results and omit the details of the analysis, but the readers may refer to [30], [31] for more details. According to this analysis, the stationary probability of a node being active, i.e. the throughput of a node, is given by

$$
\sigma=\frac{1-a}{2-a}
$$

where $a$ is the solution of

$$
f(a)=v a^{d}+a-1=0
$$

and $v$ is the call arrival rate for calls with unit mean duration. In our case, the packet lengths are not equal to one so $v=\hat{\lambda} t_{l}$ where $\hat{\lambda}$ is the normalized sensing rate and $t_{l}$ is the packet duration. Equation (16) has a unique solution since $f(0)=-1, f(1)=v>0$ and $f^{\prime}(a)>0$.

If the solution of (15) is substituted into (16), the normalized carrier-sensing rate corresponding to a given throughput can be obtained as

$$
\hat{\lambda}=\frac{(1-2 \sigma)^{-d}(1-\sigma)^{d-1} \sigma}{t_{l}}
$$

which leads to the following relationship between throughput and the carrier-sensing rate considering (1):

$$
\lambda=\frac{\sigma}{-t_{c} \sigma+t_{l}(1-2 \sigma)^{d}(1-\sigma)^{1-d}} .
$$

To have $\lambda>0$, the following condition has to be satisfied

$$
\frac{(1-2 \sigma)^{d}(1-\sigma)^{1-d}}{\sigma}>\frac{t_{c}}{t_{l}}
$$

which poses an upper bound on $\sigma$ :

$$
\sigma \leq \sigma_{d}^{\max } .
$$

Rewriting (19), $\sigma_{d}^{\max }$ is the solution to the equation:

$$
\left(\frac{1-2 \sigma_{d}^{\max }}{1-\sigma_{d}^{\max }}\right)^{d}=\frac{t_{c}}{t_{l}} \frac{\sigma_{d}^{\max }}{1-\sigma_{d}^{\max }} .
$$

For $d=2$, the maximum throughput, $\sigma_{2}^{\max }$, is given by

$$
\sigma_{2}^{\max }=\frac{1}{2}-\frac{1}{2 \sqrt{4 \frac{t_{l}}{t_{c}}+1}} .
$$

For $d>2$, we obtain lower and upper bounds on $\sigma_{d}^{\max }$, which are presented in Section 5.

Similar to the single-hop case, it is possible to obtain the mean duration between two successive transmissions by solving

$$
\frac{t_{l}}{t_{l}+E\left[T_{i}\right]}=\sigma
$$


which gives the solution:

$$
E\left[T_{i}\right]=\frac{t_{l}(1-\sigma)}{\sigma} .
$$

During an inter-transmission time, a node can be in any of three different states: It can be sleeping, carrier-sensing or receiving a packet. Since each transmitted packet has to be received by another node in the network, the total number of transmissions and receptions in the network must be the same. In the studied random regular network, each node has the same number of neighbors and has the same carriersensing rate so the behavior of nodes are symmetric. For that reason, the number of packets that a node transmits equals to the number of packets it receives on the average when the network is monitored over a long period of time:

$$
\lim _{t \rightarrow \infty} \frac{E\left[N_{r}(t)\right]}{E\left[N_{t}(t)\right]}=1,
$$

where $N_{t}(t)$ and $N_{r}(t)$ correspond to the number of packets that a node transmits and receives within a time period $t$, respectively. Since there is a transmission at the beginning of each inter-transmission time, i.e., there are $N_{t}(t)-1$ inter-transmission periods within a duration of $t$, the expected time spent in receiving a packet during an inter-transmission time equals to the transmission duration of one packet:

$$
E\left[T_{r}\right]=\lim _{t \rightarrow \infty} \frac{E\left[N_{r}(t)\right] t_{l}}{E\left[N_{t}(t)\right]-1}=t_{l} .
$$

However, it should be noted that this is an average behavior, there can be more than one receptions or no receptions during a specific inter-transmission time. Remaining time of the inter-transmission duration is shared between the time spent for carrier-sensing and time spent for sleeping. Time spent for sleeping can be written as

$$
E\left[T_{s}\right]=\left(E\left[T_{i}\right]-E\left[T_{r}\right]\right) \frac{\frac{1}{\lambda}}{\frac{1}{\lambda}+t_{c}}=\frac{t_{l}(1-2 \sigma)}{\sigma} \frac{\frac{1}{\lambda}}{\frac{1}{\lambda}+t_{c}} .
$$

Using the relationship between $\lambda$ and $\sigma$ given by (18), $E\left[T_{S}\right]$ can be obtained only in terms of $\sigma$ as

$$
E\left[T_{s}\right]=\frac{t_{l}-3 t_{l} \sigma-t_{c}(1-2 \sigma)^{1-d}(1-\sigma)^{d} \sigma+2 t_{l} \sigma^{2}}{\sigma-\sigma^{2}} .
$$

Time spent for carrier-sensing can similarly be written as

$$
\begin{aligned}
E\left[T_{c}\right] & =\left(E\left[T_{i}\right]-E\left[T_{r}\right]\right) \frac{t_{c}}{\frac{1}{\lambda}+t_{c}} \\
& =t_{c}(1-2 \sigma)^{1-d}(1-\sigma)^{d-1} .
\end{aligned}
$$

Then, total energy consumption per packet is given by

$$
\begin{aligned}
E\left[E_{p}\right]= & E\left[T_{s}\right] P_{s}+E\left[T_{c}\right] P_{c}+E\left[T_{t}\right] P_{t}+E\left[T_{r}\right] P_{r} \\
= & t_{l}\left(P_{r}+P_{t}+P_{s}\left(-2+\frac{1}{\sigma}\right)\right)+ \\
& \left(P_{c}-P_{s}\right) t_{c}(1-2 \sigma)^{1-d}(1-\sigma)^{-1+d}
\end{aligned}
$$

and the energy per transmitted bit is given by

$$
E\left[E_{b}\right]=\frac{E\left[E_{p}\right]}{t_{l} R} .
$$

The energy-optimum throughput, $\sigma_{d}^{*}$, which minimizes $E\left[E_{b}\right]$ can be found algebraically by solving $\frac{\partial E\left[E_{b}\right]}{\partial \sigma}=0$ as given by

$$
(d-1)\left(P_{c}-P_{s}\right) t_{c}(1-2 \sigma)^{-d}(1-\sigma)^{d-2}-\frac{P_{s} t_{l}}{\sigma^{2}}=0 .
$$

The solution for $d=2$ can be found as

$$
\sigma_{2}^{*}=\frac{1}{2+\sqrt{\frac{\left(P_{c}-P_{s}\right) t_{c}}{P_{s} t_{l}}}} .
$$

For $d=3$ and $d=4$, it is also possible to obtain a close form expression for $\sigma_{d}^{*}$ but we do not present these results here due to space constraints. For $d \geq 5$, a numerical solution has to be obtained but we provide several bounds for the optimum throughput in the next section. The corresponding energy-optimum carrier-sensing rate for $d=2$ can be found by substituting (35) into (18) as:

$$
\lambda_{2}^{*}=\frac{t_{l}+\sqrt{\left(\frac{P_{c}}{P_{s}}-1\right) t_{c} t_{l}}}{t_{c}\left(t_{l}\left(\frac{P_{c}}{P_{s}}-2\right)-\sqrt{t_{c} t_{l}\left(\frac{P_{c}}{P_{s}}-1\right)}\right)}
$$

for $\sigma_{2}^{*} \leq \sigma_{2}^{\max }$.

\section{BOUNDS ON THE ENERGY-OPTIMUM ThROUGHPUT AND MAXIMUM THROUGHPUT}

The exact solution of the maximum throughput and the energy-optimum throughput are presented only for the $d=$ 2 case. In this part, we obtain lower and upper bounds on the maximum throughput, $\sigma_{d}^{\max }$, and the energy-optimum throughput, $\sigma_{d}^{*}$ where $\sigma_{d}^{\max }$ is the solution to (21) and $\sigma_{d}^{*}$ is the solution to (34).

\subsection{Lower Bounds on the Maximum Throughput, $\sigma_{d}^{\max }$}

Since $\sigma_{d}^{\max }<\frac{1}{2}$, right hand side of (21) can be bounded as

$$
\left(\frac{1-2 \sigma_{d}^{\max }}{1-\sigma_{d}^{\max }}\right)^{d}=\frac{t_{c}}{t_{l}} \frac{\sigma_{d}^{\max }}{1-\sigma_{d}^{\max }} \leq \frac{t_{c}}{t_{l}}
$$

giving the following lower bound:

$$
\sigma_{d}^{\max } \geq \frac{1-\left(\frac{t_{c}}{t_{l}}\right)^{1 / d}}{2-\left(\frac{t_{c}}{t_{l}}\right)^{1 / d}} \triangleq \underline{\sigma}_{d}^{\max , 1} .
$$

Another lower bound can be found by rewriting (21) as

$$
\left(1-2 \sigma_{d}^{\max }\right)=f\left(\sigma_{d}^{\max }, d\right)\left(\frac{t_{c}}{t_{l}}\right)^{1 / d},
$$

where

$$
f\left(\sigma_{d}^{\max }, d\right)=\left(1-\sigma_{d}^{\max }\right)\left(\frac{\sigma_{d}^{\max }}{1-\sigma_{d}^{\max }}\right)^{1 / d} .
$$

For $0<\sigma_{d}^{\max }<1, f\left(\sigma_{d}^{\max }, d\right)$ has a single maximum at $\sigma_{d}^{\max }=\frac{1}{d}$ since $f^{\prime}>0$ if $\sigma_{d}^{\max }<\frac{1}{d}$ and $f^{\prime}<0$ if $\sigma_{d}^{\max }>\frac{1}{d}$. Hence,

$$
\left(1-2 \sigma_{d}^{\max }\right) \leq\left(1-\frac{1}{d}\right)\left(\frac{\frac{1}{d}}{1-\frac{1}{d}}\right)^{1 / d}\left(\frac{t_{c}}{t_{l}}\right)^{1 / d}
$$


which gives the following lower bound:

$$
\sigma_{d}^{\max } \geq \frac{1}{2}-\frac{(d-1)^{\left(1-\frac{1}{d}\right)}}{2 d}\left(\frac{t_{c}}{t_{l}}\right)^{1 / d} \triangleq \underline{\sigma}_{d}^{\max , 2} .
$$

\subsection{Upper Bound on the Maximum Throughput, $\sigma_{d}^{\max }$}

An upper bound on $\sigma_{d}^{\max }$ can be found using an approximation of (21) as $\sigma_{d}^{\max } \rightarrow \frac{1}{2}$ :

$$
\left(\frac{1-2 \sigma_{d}^{\max }}{1-\sigma_{d}^{\max }}\right)^{d}=\frac{t_{c}}{t_{l}} \frac{\sigma_{d}^{\max }}{1-\sigma_{d}^{\max }} \approx \frac{t_{c}}{t_{l}}
$$

which can be written as

$$
1-2 \sigma_{d}^{\max } \approx\left(1-\sigma_{d}^{\max }\right)\left(\frac{t_{c}}{t_{l}}\right)^{\frac{1}{d}} .
$$

Since $\left(1-\sigma_{d}^{\max }\right)>\frac{1}{2}$, an approximate upper bound on $\sigma_{d}^{\max }$ is given by

$$
\sigma_{d}^{\max } \lesssim \frac{1}{2}-\frac{1}{4}\left(\frac{t_{c}}{t_{l}}\right)^{\frac{1}{d}} \triangleq \bar{\sigma}_{d}^{\max } .
$$

It is should be noted that the bound gets tighter as $\sigma_{d}^{\max } \rightarrow \frac{1}{2}$.

\subsection{Lower Bound on the Energy-Optimum Throughput, $\sigma_{d}^{*}$}

(34) can be rewritten as

$$
1-2 \sigma_{d}^{*}=g\left(\sigma_{d}^{*}, d\right)\left(\frac{\left(P_{c}-P_{s}\right) t_{c}(d-1)}{P_{s} t_{l}}\right)^{1 / d}
$$

where

$$
g\left(\sigma_{d}^{*}, d\right)=\left(\frac{\sigma_{d}^{*}}{1-\sigma_{d}^{*}}\right)^{2 / d}\left(1-\sigma_{d}^{*}\right) .
$$

Since $g^{\prime}>0$ if $\sigma_{d}^{*}<\frac{2}{d}$ and $g^{\prime}<0$ if $\sigma_{d}^{*}>\frac{2}{d}$ for $0<\sigma_{d}^{*}<1, g$ has a single maximum at $\sigma_{d}^{*}=\frac{2}{d}$. Then, an inequality can be written as

$$
\begin{aligned}
& 1-2 \sigma_{d}^{*} \leq \\
& \left(\frac{\frac{2}{d}}{1-\frac{2}{d}}\right)^{2 / d}\left(1-\frac{2}{d}\right)\left(\frac{\left(P_{c}-P_{s}\right) t_{c}(d-1)}{P_{s} t_{l}}\right)^{1 / d}
\end{aligned}
$$

which gives the following lower bound:

$$
\sigma_{d}^{*} \geq \frac{1}{2}-\frac{(d-2)^{\left(1-\frac{2}{d}\right)}\left(\frac{(d-1)\left(P_{c}-P_{s}\right) t_{c}}{P_{s} t_{l}}\right)^{\frac{1}{d}}}{d\left(2^{1-\frac{2}{d}}\right)} \triangleq \underline{\sigma}_{d}^{*} .
$$

\subsection{Upper Bound on the Energy-Optimum Throughput, $\sigma_{d}^{*}$}

It is possible to write (34) as a fixed point equation which can be bounded as given by:

$$
\begin{aligned}
\sigma_{d}^{*}= & \left(\frac{1-2 \sigma_{d}^{*}}{1-\sigma_{d}^{*}}\right)^{\frac{d}{2}}\left(1-\sigma_{d}^{*}\right)\left(\sqrt{\frac{\left(P_{c}-P_{s}\right) t_{c}}{P_{s} t_{l}}(d-1)}\right)^{-1} \\
& \leq\left(1-\sigma_{d}^{*}\right)\left(\sqrt{\frac{\left(P_{c}-P_{s}\right) t_{c}}{P_{s} t_{l}}(d-1)}\right)^{-1}
\end{aligned}
$$

which gives an upper bound on $\sigma_{d}^{*}$ :

$$
\sigma_{d}^{*} \leq\left(\sqrt{\frac{(d-1)\left(P_{c}-P_{s}\right) t_{c}}{P_{s} t_{l}}}+1\right)^{-1} \triangleq \bar{\sigma}_{d}^{*} .
$$

\subsection{Lower Bound on $\sigma_{d}^{*} / \sigma_{d}^{\max }$}

A lower bound on the ratio $\sigma_{d}^{*} / \sigma_{d}^{\max }$ can be obtained by dividing the lower bound for $\sigma_{d}^{*}$ by the upper bound for $\sigma_{d}^{\max }$ :

$$
\begin{aligned}
\frac{\sigma_{d}^{*}}{\sigma_{d}^{\max }} \gtrsim \frac{\underline{\sigma}_{d}^{*}}{\bar{\sigma}_{d}^{\max }}= \\
\frac{2\left(d-4^{\left.\frac{1}{d}(d-2)^{1-\frac{2}{d}}\left(\frac{(d-1) t_{c}\left(P_{c}-P_{s}\right)}{P_{s} t_{l}}\right)^{1 / d}\right)}\right.}{\left(2-\left(\frac{t_{c}}{t l}\right)^{1 / d}\right) d} .
\end{aligned}
$$

\subsection{Upper Bound on $\sigma_{d}^{*} / \sigma_{d}^{\max }$}

Dividing $\bar{\sigma}_{d}^{*}$ by $\underline{\sigma}_{d}^{\max , 1}$, an upper bound on the ratio $\sigma_{d}^{*} / \sigma_{d}^{\max }$ can be obtained as:

$$
\begin{aligned}
\frac{\sigma_{d}^{*}}{\sigma_{d}^{\max }} & \leq \frac{\bar{\sigma}_{d}^{*}}{\sigma_{d}^{\max , 1}}= \\
& \left(\sqrt{\frac{(d-1)\left(P_{c}-P_{s}\right) t_{c}}{P_{s} t_{l}}}+1\right)^{-1} \frac{2-\left(\frac{t_{c}}{t_{l}}\right)^{1 / d}}{1-\left(\frac{t_{c}}{t_{l}}\right)^{1 / d}} .
\end{aligned}
$$
bound:

Similarly, dividing $\bar{\sigma}_{d}^{*}$ to $\underline{\sigma}_{d}^{\max , 2}$ gives another upper

$$
\frac{\sigma_{d}^{*}}{\sigma_{d}^{\max }} \leq \frac{\bar{\sigma}_{d}^{*}}{\underline{\sigma}_{d}^{\max , 2}}=\frac{\left(\sqrt{\frac{(d-1)\left(P_{c}-P_{s}\right) t_{c}}{P_{s} t_{l}}}+1\right)^{-1}}{\frac{1}{2}-\frac{(d-1)^{\left(1-\frac{1}{d}\right)}}{2 d}\left(\frac{t_{c}}{t_{l}}\right)^{1 / d}} .
$$

\section{Numerical Results}

\subsection{Single-Hop Network}

We first investigate the accuracy of the proposed energy consumption analysis for the single-hop case. We performed simulations for $N=5,10$ and 100. Simulation parameters are based on the measurements from the Mica2 mote reported in [1]: $P_{t}=60 \mathrm{~mW}, P_{c}=P_{r}=45 \mathrm{~mW}$, $P_{s}=0.09 \mathrm{~mW}, t_{l}=15 \mathrm{~ms}, t_{c}=0.35 \mathrm{~ms}$ and $R=19.23 \mathrm{~Kb} / \mathrm{s}$. For each $N$, we performed simulations by increasing $\lambda$ and we recorded the corresponding throughput and energy consumption in the network.

Fig. 5(a) presents the total energy consumption as the total throughput in the network increases. Figure also depicts (11) versus $N \sigma$ which matches with the simulation results. The two components of energy consumption, energy consumed while sleeping and carrier-sensing, are plotted in Fig. 5(b) and (c), respectively. The high accuracy of the match between simulation and analytical results shows that the assumption of exponentially distributed carrier-sensing durations does not affect the accuracy of the analysis.

From Fig. 5(a), it can be observed that the energy consumption is high at both ends of the throughput axis. At low throughputs, the energy consumed while sleeping 


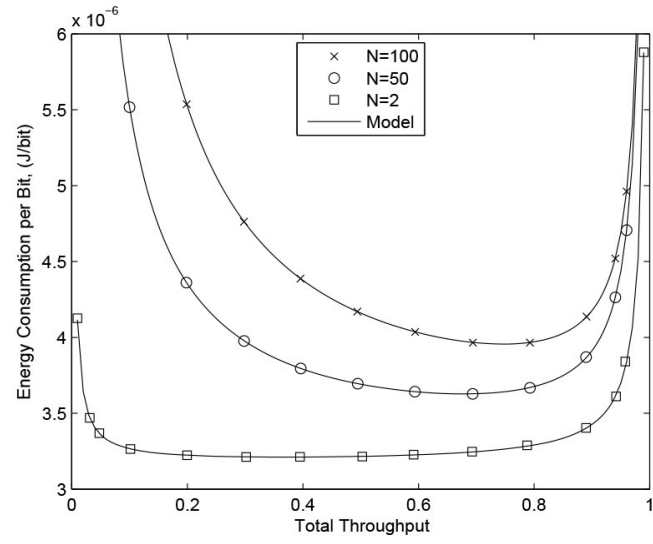

(a)

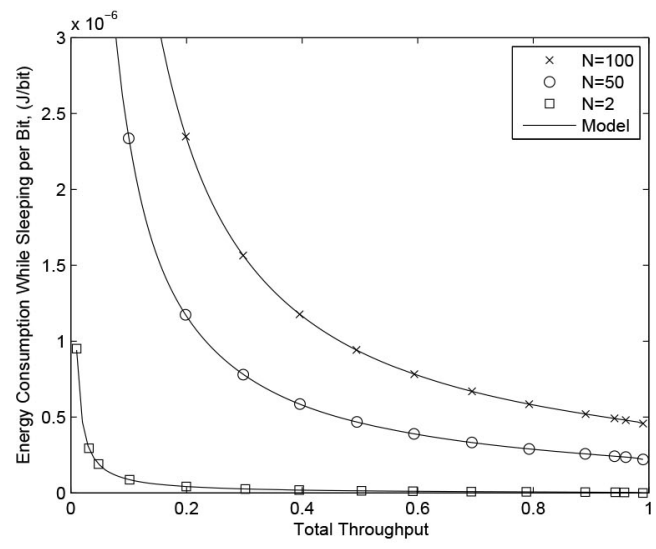

(b)

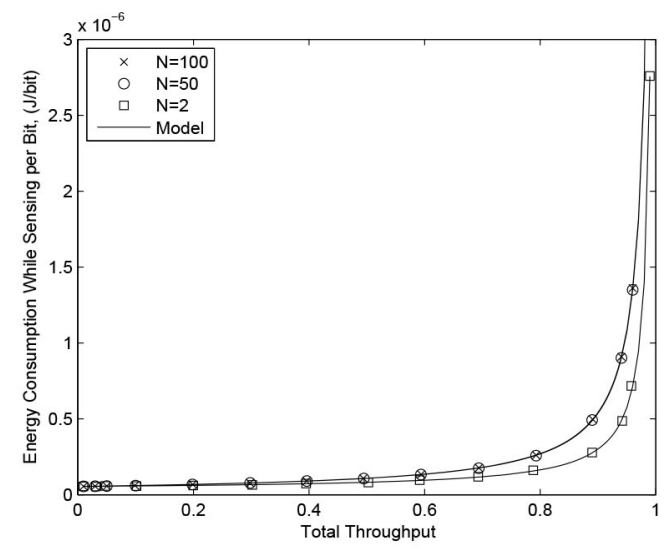

(c)

Fig. 5. Energy consumption per node in the single-hop network. (a) Total energy consumption. (b) Energy consumed while sleeping. (c) Energy consumed while carrier sensing.

(Fig. 5(b)) dominates the total energy consumption whereas energy consumed while carrier-sensing (Fig. 5(c)) dominates the total energy consumption at high throughputs.

It can be observed that the energy consumption per bit is higher for networks with a larger number of nodes. The main reason of this increase is associated with the increased sleeping costs with $N$ as it can be seen in Fig. 5(b). In a single-hop network, only a single node can transmit at a

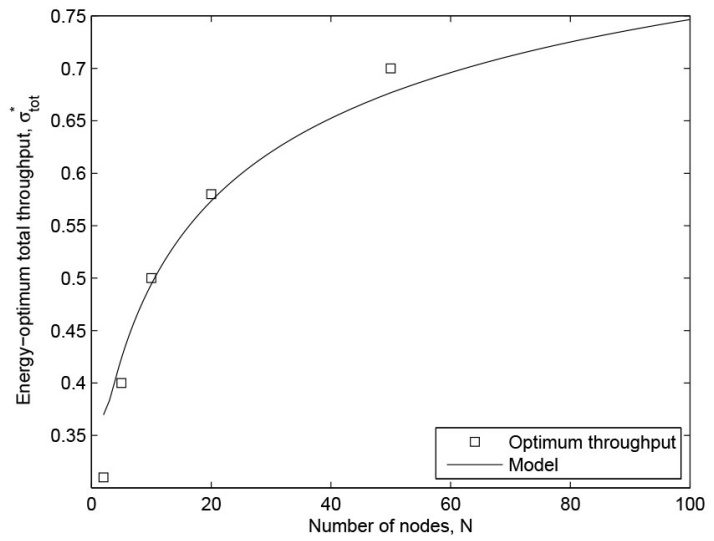

Fig. 6. Change of energy-optimum total throughput as the number of nodes increases for the single-hop network.

time so the rest of the nodes are sleeping. This results in an approximately linear increase in the sleeping costs with $N$ so total energy consumption increases with $N$.

It can also be observed that the energy-optimum total throughput increases as $N$ increases. Fig. 6 plots the energy-optimum total throughput as the number of nodes increases along with the proposed optimum throughput given by (14). The reason behind this increase is the different behaviors of energy consumed while sleeping and carrier-sensing as the number of nodes increases. The energy consumed while sleeping increases approximately linearly with the number of nodes. On the other hand, the energy consumed for carrier-sensing does not increase significantly with the number of nodes as it can be observed from Fig. 5(c). So, the trade-off throughput tends to increase as $N$ increases since the sleeping costs are lower at high throughputs.

Fig. 7 plots the optimum carrier-sensing frequency per node as the number of nodes increases. The figure also depicts (13) obtained from the analytical model. The model predicts the optimum carrier-sensing rate per node very accurately. To achieve energy minimization per bit, the nodes should reduce their carrier-sensing frequency approximately in proportional to $1 / \sqrt{N}$ as it can be deduced from (13).

Figs. 8 and 9 depict the energy-optimum carrier-sensing rate and energy-optimum throughput as the ratio of

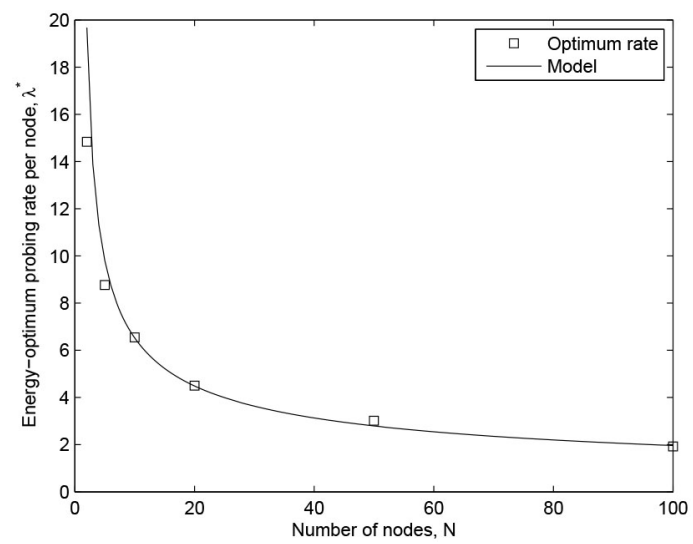

Fig. 7. Energy-optimum carrier-sensing rate per node as the number of nodes increases for the single-hop network. 


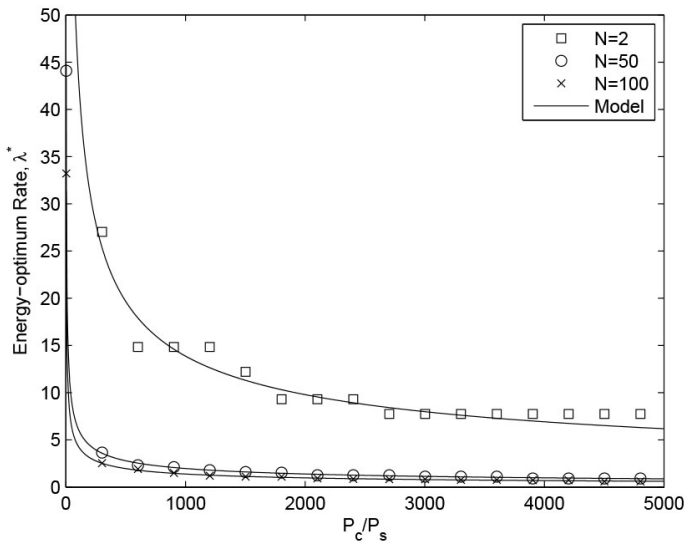

Fig. 8. Energy-optimum carrier-sensing rate per node as $P_{C} / P_{S}$ increases for the single-hop network.

$P_{c} / P_{s}$ changes, respectively. As the cost of carrier-sensing increases with respect to sleeping, the nodes need to sense the channel less frequently to minimize energy consumption per bit, so the energy-optimum rate and throughput reduces.

\subsection{Multi-Hop Network}

To evaluate our analytical model for multi-hop networks, we performed simulations for random regular conflict graphs with $d=2,3$ and 10, which are created by the topology generation algorithm proposed by Viger [32]. Each simulated conflict graph consists of 1000 nodes.

We first investigate the accuracy of the relationship between the carrier sensing rate and the throughput given by (15) and (16) for random regular conflict graphs. Although the analysis is for a Cayley tree conflict graph where each internal node has a degree of $d$, we performed simulations for both the tree conflict graph and the random regular conflict graphs where each node has a degree of $d$ for a unit packet length. As it can be seen from Fig. 10, the analysis is highly accurate for random regular conflict graphs as well as the Cayley-tree conflict graph. This result suggests that the relationship between the throughput and the carrier sensing rate mainly depends on the degree of the conflict graph.

We now investigate the energy consumption of the multi-hop network with the same parameters as the

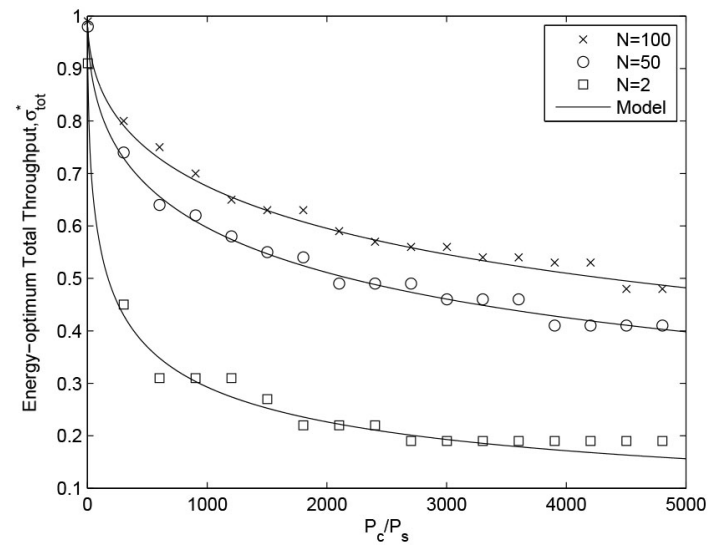

Fig. 9. Energy-optimum total throughput as $P_{C} / P_{S}$ increases for the single-hop network.

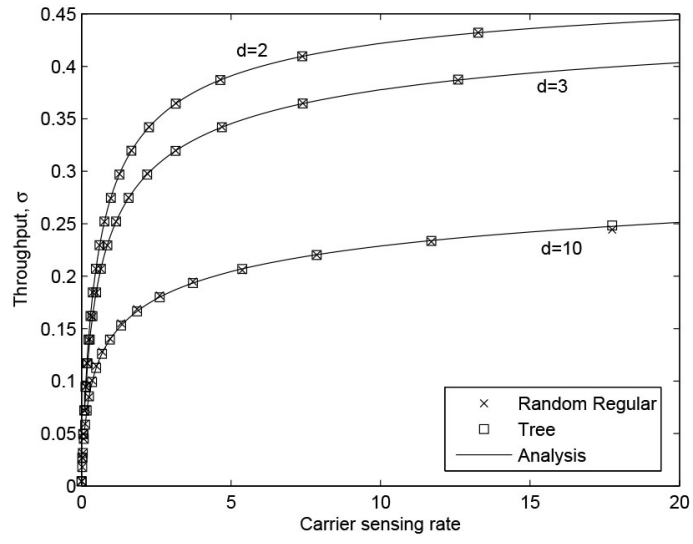

Fig. 10. Relationship between the throughput and the carrier sensing rate for tree conflict graphs and random regular conflict graphs with $d=2,3$ and 4 .

single-hop case as given in Section 6.1. The average energy consumption of the network per transmitted bit and the components of the energy consumption are shown in Fig. 11 for $d=2,3$ and 10 along with the values obtained from the proposed analytical model as given by (33). At low throughputs, sleeping increases the energy consumption per transmitted bit, and at high throughputs, the energy spent for carrier sensing dominates. As $d$ increases, the energy spent for carrier sensing becomes significant because the probability that a carrier sensing attempt fails increases due to higher interference.

Fig. 12 plots how the energy-optimum carrier sensing rate changes as a function of $P_{c} / P_{s}$. As the energy consumption for carrier sensing increases, the energy-optimum carrier sensing rate reduces. Each failed carrier sensing attempt wastes energy-if carrier sensing is very expensive, nodes need to be less aggressive in order to reduce the probability of finding the channel busy. Fig. 13 plots the corresponding energy-optimum throughput obtained. For $d=2$, (36) and (35) closely match with the energy-optimum carrier sensing rate and the energy-optimum throughput. For $d=3$ and $d=10$, the numerical solution of (34) is used to obtain the energy-optimum throughput and the result is substituted into (18) to obtain the energy-optimum carrier-sensing rate.

\subsection{Bounds on the $\sigma_{d}^{\max }$ and $\sigma_{d}^{*}$ for the Multi-Hop Network}

In this part, we demonstrate the change in the $\sigma_{d}^{*}$ and $\sigma_{d}^{\max }$ with $d$ and evaluate the performance of the proposed bounds. Fig. 14 plots $\sigma_{d}^{\max }$ as $d$ increases for $\frac{t_{c}}{t_{1}} \approx 0.02$ which corresponds to the simulation parameters used in this section and for $\frac{t_{c}}{t_{l}}=0.001$ which is the case where carrier-sensing takes a shorter time in comparison to the packet duration. In this figure, the lower and upper bounds on $\sigma_{d}^{\max }$ derived in Section 5.1 and Section 5.2 are also depicted. At low degrees, $\underline{\sigma}_{d}^{\max , 2}$ provides a better lower bound but $\underline{\sigma}_{d}^{\max , 1}$ performs better at higher degrees. On the other hand, the upper bound $\bar{\sigma}_{d}^{\max }$ is tight for small values of $d$ but it becomes looser as $d$ increases. One of the reasons behind this behavior is that the approximation made in the derivation of $\bar{\sigma}_{d}^{\max }$ gets more accurate as $\sigma_{d}^{\max } \rightarrow \frac{1}{2}$. 


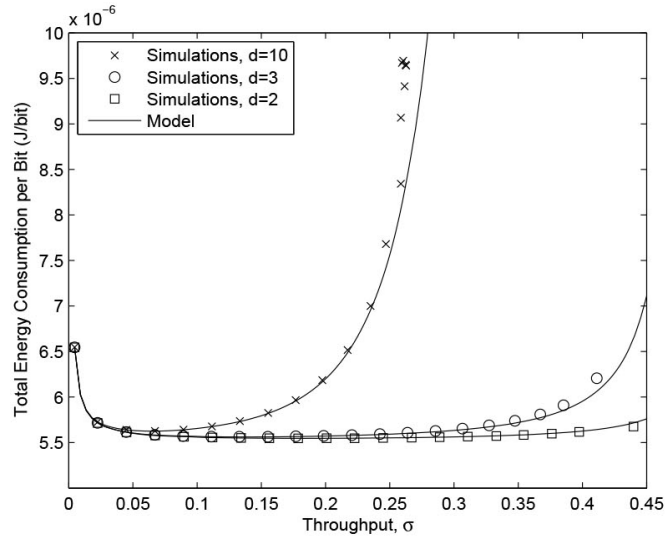

(a)

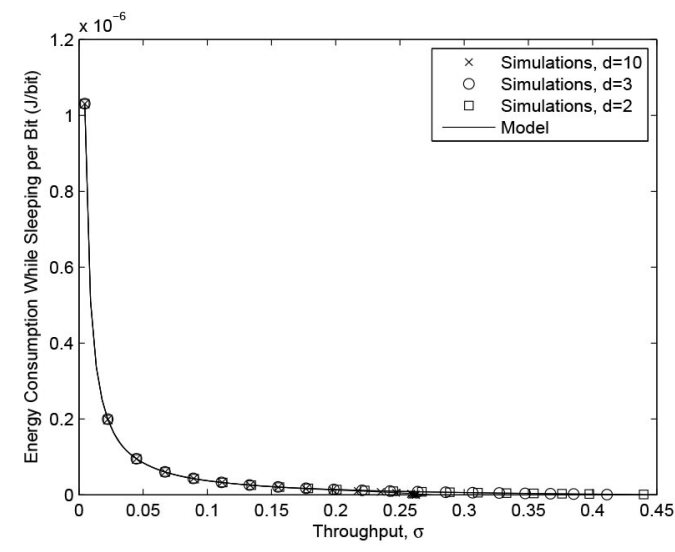

(b)

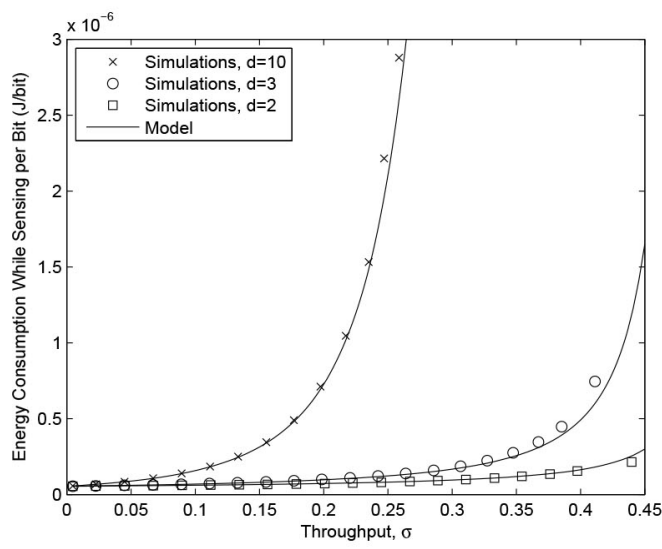

(c)

Fig. 11. Energy consumption per node in the multi-hop network. (a) Total energy consumption. (b) Energy consumed while sleeping. (c) Energy consumed while carrier sensing

Fig. 15 plots the energy-optimum throughput, $\sigma_{d}^{*}$, along with its lower and upper bounds. For $\frac{t_{c}}{t_{l}} \approx 0.02, \underline{\sigma}_{d}^{*}$ results in negative values for $d<8$ but its tightness improves as $d$ increases. For $\frac{t_{c}}{t_{l}}=0.001, \underline{\sigma}_{d}^{*}$ provides a very tight bound by differing less than $0.1 \%$ from $\sigma_{d}^{*}$ at $d=20$. The upper bound $\bar{\sigma}_{d}^{*}$ is loose for $\frac{t_{c}}{t_{l}}=0.001$, however, it provides an upper bound which changes nearly parallel to $\sigma_{d}^{*}$ for $\frac{t_{c}}{t_{l}}=0.02$ for the considered range of $d$ values.

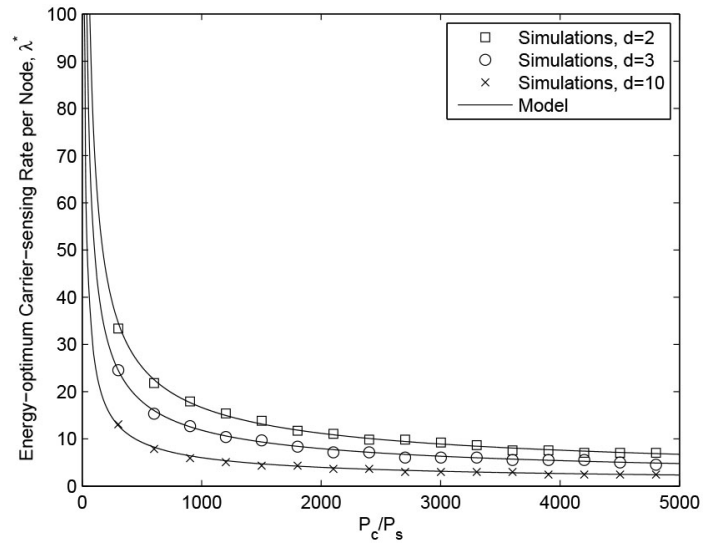

Fig. 12. Energy-optimum carrier sensing rate as a function of $\frac{P_{C}}{P_{S}}$ for the multi-hop network.

The ratio of the energy-optimum throughput to the maximum throughput is plotted in Fig. 16 along with the lower and upper bounds $\frac{\sigma_{d}^{*}}{\bar{\sigma}_{d}^{\max }}, \frac{\bar{\sigma}_{d}^{*}}{\sigma_{d}^{\max , 1}}$ and $\frac{\bar{\sigma}_{d}^{*}}{\sigma_{d}^{\max , 2}}$. It is observed that the ratio $\frac{\sigma_{d}^{*}}{\sigma_{d}^{\max }}$ decreases as $d$ increases. For $\frac{t_{c}}{t_{l}}=0.001$, the upper bounds are loose. Since the energy-optimum throughput cannot exceed the maximum throughput, only values less than one are depicted in Fig. 16(b). However, for $\frac{t_{c}}{t_{l}} \approx 0.02$, the upper bounds demonstrate that the energyoptimum throughput cannot exceed half of the maximum throughput for $d \leq 20$.

\subsection{Comparison of Non-Persistent CSMA With 1-Persistent CSMA}

In this part, we compare the non-persistent CSMA with 1-persistent CSMA in terms of energy consumption. In the non-persistent CSMA, a node sleeps for a random amount when it finds the channel busy during its transmission attempt. In the 1-persistent CSMA, however, a node continues to sense the channel if it finds the channel busy [3]. The node immediately transmits its packet as soon as the channel becomes idle.

We performed simulations to compare the energy consumption of both protocols. To do a fair comparison, we have implemented the zero-collision assumption that we have incorporated for the non-persistent CSMA in the simulations of the 1-persistent CSMA: If more than one node

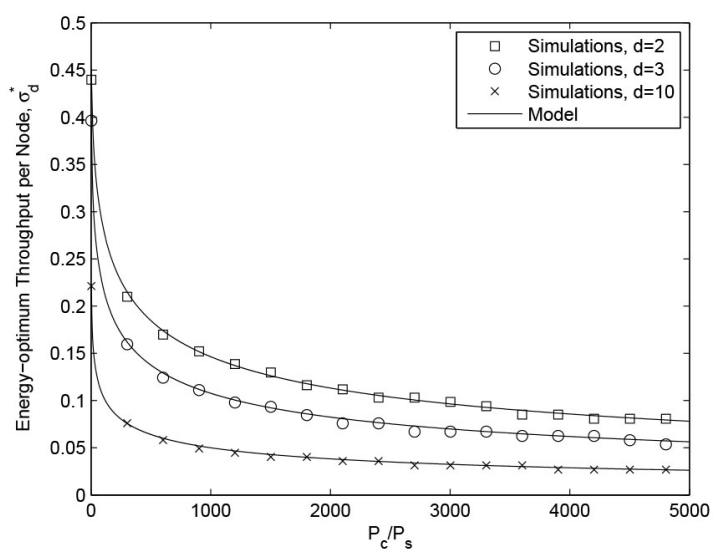

Fig. 13. Energy-optimum throughput as a function of $\frac{P_{C}}{P_{S}}$ for the multihop network. 


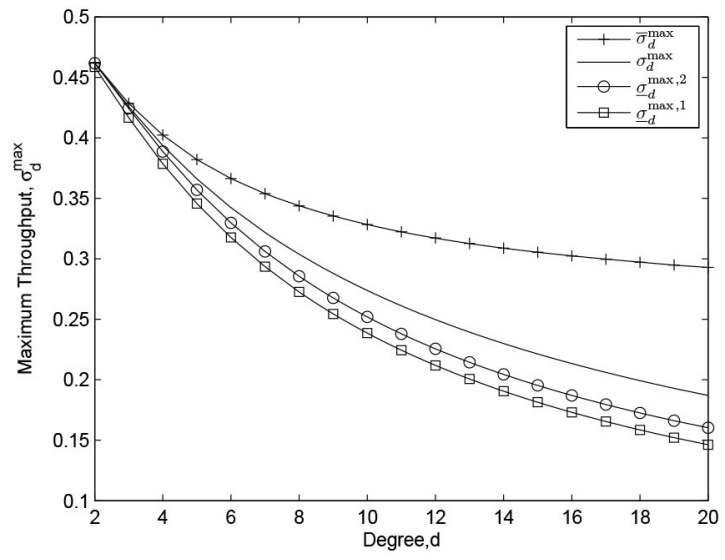

(a)

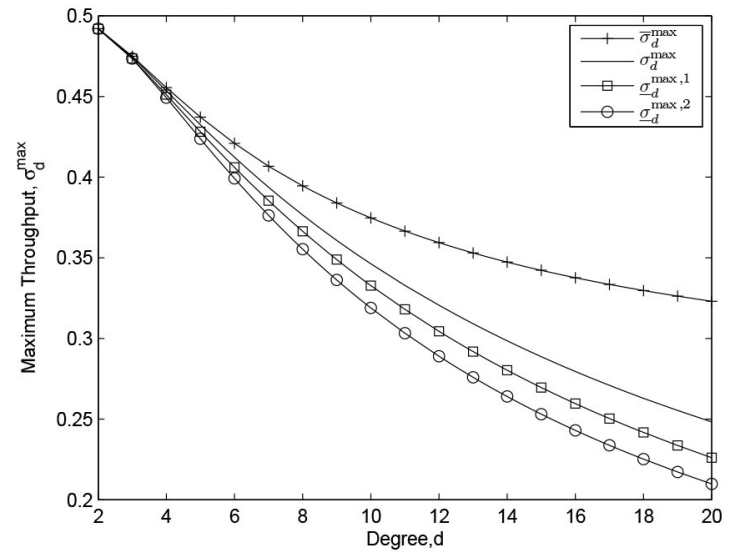

(b)

Fig. 14. Maximum throughput as a function of $d$ for the multi-hop network for (a) $\frac{t_{c}}{t_{l}} \approx 0.02$, and (b) $\frac{t_{c}}{t_{l}}=0.001$.

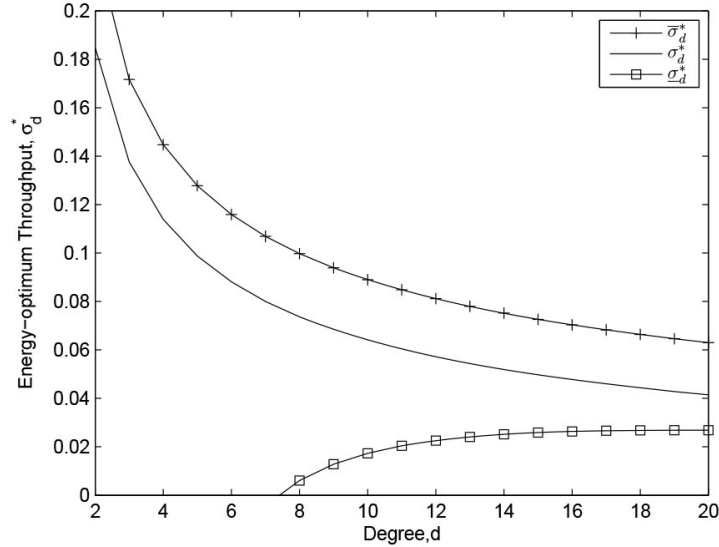

(a)

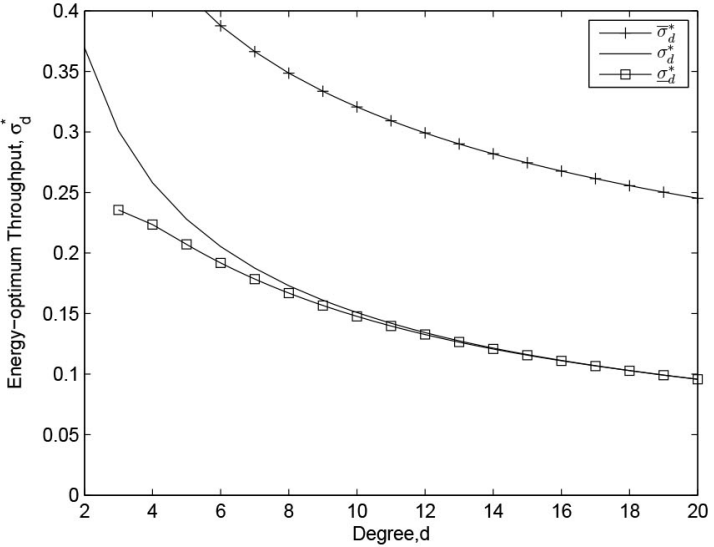

(b)

Fig. 15. Energy-optimum throughput as a function of $d$ for the multi-hop network for (a) $\frac{t_{C}}{t_{l}} \approx 0.02$, and (b) $\frac{t_{C}}{t_{l}}=0.001$.

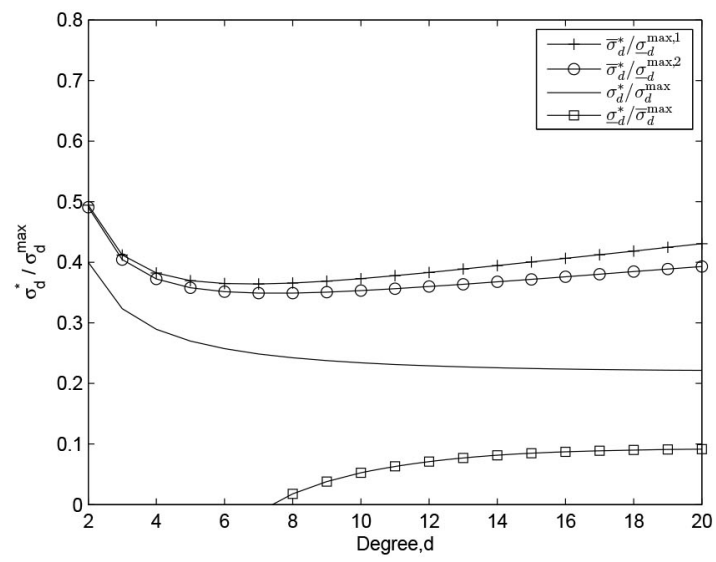

(a)

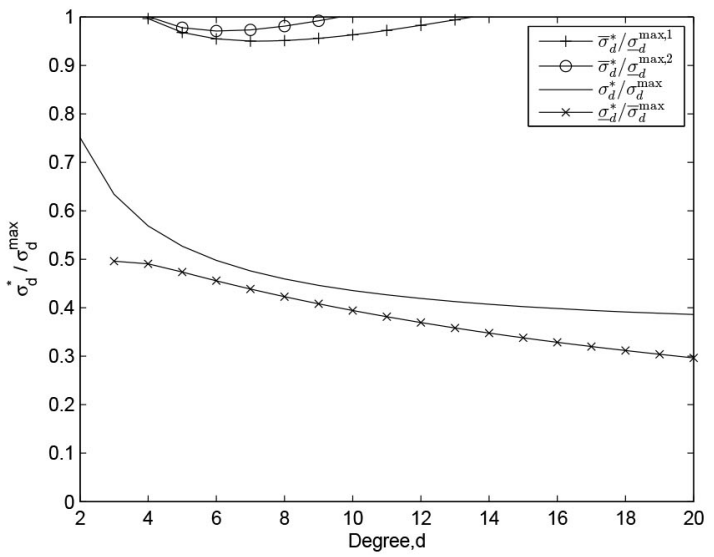

(b)

Fig. 16. Ratio of energy-optimum throughput to maximum throughput as a function of $d$ for the multi-hop network for $(\mathrm{a}) \frac{t_{c}}{t_{l}} \approx 0.02$, and (b) $\frac{t_{C}}{t_{l}}=0.001$.

is waiting for the channel to become available to transmit a packet, we have allowed only one of the nodes to transmit its packet so that a collision is prevented while remaining nodes continue to sense the channel.
Fig. 17 presents the energy consumption of both protocols as a function of throughput for a single-hop scenario with 50 nodes. At low throughputs, the energy consumption of both protocols do not differ significantly 


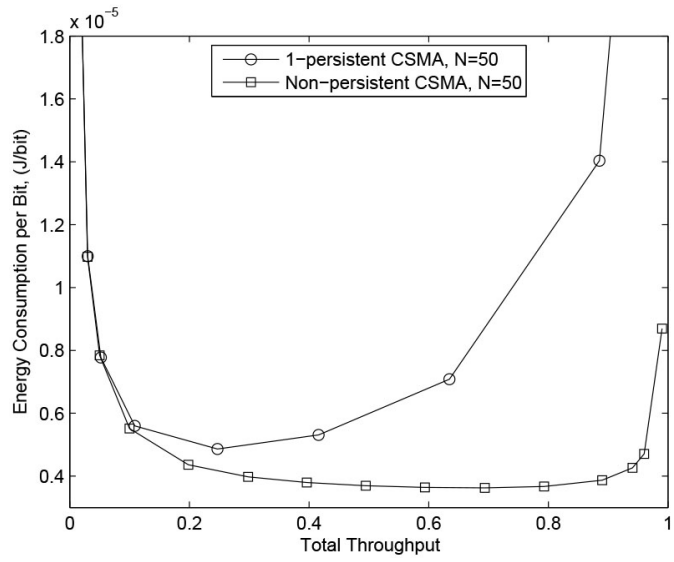

Fig. 17. Energy consumption comparison of 1-persistent CSMA versus non-persistent CSMA for the single-hop scenario.

because nodes do not frequently find the channel busy in both cases. So, both protocols behave in a similar fashion which results in a similar energy consumption. However, as the throughput increases, energy consumption of the 1-persistent CSMA protocol significantly increases because nodes frequently find the channel busy and continue to listen the channel to capture it when it becomes idle. In the non-persistent scenario, on the other hand, the nodes go to sleep when they find the channel busy. Since the energy consumption of channel sensing is very high in comparison to sleeping, the energy consumption of 1-persistent CSMA is higher than non-persistent CSMA at higher throughputs. For that reason, non-persistent CSMA should be preferred for battery-limited wireless applications.

\section{CONCLUSION}

We proposed an energy consumption model of a node in a CSMA network. The proposed model shows that the number of failed carrier sensing attempts significantly increases at high throughputs causing energy waste. On the contrary, at low throughputs, nodes sleep during most of their lifetimes which also results in energy waste as far as the energy per transmitted bit is considered. We derived the energy-optimum carrier sensing rate and the corresponding energy-optimum throughput for both a single-hop network and a multi-hop network.

For single-hop networks, we observe that the energyoptimum throughput increases with the number of nodes sharing the channel. On the other hand, the energyoptimum throughput reduces with the degree of the conflict graph for multi-hop networks. For both the single-hop and multi-hop case, our results suggest that as the power required for carrier sensing increases, the energy-optimum sensing rate and throughput reduce. By proposing several bounds, we show that the energy-optimum throughput cannot exceed approximately half of the maximum throughput for the simulation parameters obtained from Mica2 measurements.

Our results have implications for the design of adaptive optimal-CSMA algorithms. We observe a dramatic increase in the carrier-sensing rate as the throughput approaches its limit, as a result, the energy consumption also increases significantly. The trade-off between the energy consumption and throughput has to be considered in the design of adaptive MAC algorithms.

\section{ACKNOWLEDGMENTS}

This work was supported by the European Commission in the framework of the FP7 Network of Excellence in Wireless Communications NEWCOM\#.

\section{REFERENCES}

[1] J. Polastre, J. Hill, and D. Culler, "Versatile low power media access for wireless sensor networks," in Proc. 2nd Int. Conf. Embedded Networked Sensor Systems, Baltimore, MD, USA, 2004.

[2] S.-Y. Yun, Y. Yi, J. Shin, and D. Y. Eun, "Optimal CSMA: A survey," in Proc. IEEE ICCS, Nov. 2012, pp. 199-204.

[3] L. Kleinrock and F. Tobagi, "Packet switching in radio channels: Part I-carrier sense multiple-access modes and their throughputdelay characteristics," IEEE Trans. Commun., vol. 23, no. 12, pp. 1400-1416, Dec. 1975.

[4] L. Bononi, M. Conti, and L. Donatiello, "A distributed mechanism for power saving in IEEE 802.11 wireless LANs," Mobile Netw. Appl., vol. 6, no. 3, pp. 211-222, Jun. 2001.

[5] R. Bruno, M. Conti, and E. Gregori, "Optimization of efficiency and energy consumption in p-persistent CSMA-based wireless LANs," IEEE Trans. Mobile Comput., vol. 1, no. 1, pp. 10-31, Jan./Mar. 2002.

[6] P. Serrano, A. Garcia-Saavedra, M. Hollick, and A. Banchs, "On the energy efficiency of IEEE 802.11 WLANs," in Proc. EW Conf., Lucca, Italy, 2010, pp. 932-939.

[7] C. Aydogdu and E. Karasan, "An analysis of IEEE 802.11 DCF and its application to energy-efficient relaying in multihop wireless networks," IEEE Trans. Mobile Comput., vol. 10, no. 10, pp. 1361-1373, Oct. 2011.

[8] D. Chan, T. Berger, and R. Bridgelall, "Energy efficiency of CSMA protocols for wireless packet switched networks," in Proc. IEEE Wireless Communications and Networking Conf., 2004.

[9] T. Park, T. Kim, J. Choi, S. Choi, and W. Kwon, "Throughput and energy consumption analysis of IEEE 802.15.4 slotted CSMA/CA," Electron. Lett., vol. 41, no. 18, pp. 1017-1019, Sept. 2005.

[10] Y. Zhang, P. Xu, G. Bi, and F. Sheng Bao, "Analysis of energy efficiency and power saving in IEEE 802.15.4," in Proc. IEEE Wireless Communications and Networking Conf., Kowloon, Hong Kong, Mar. 2007, pp. 3330-3334.

[11] K. Ashrafuzzaman, "Energy and throughput optimal operating region in slotted CSMA/CA based WSN," IEEE Commun. Lett., vol. 16, no. 9, pp. 1524-1527, Sept. 2012.

[12] K. Langendoen and G. Halkes, "Energy-efficient medium access control," in Embedded Systems Handbook. Hoboken, NJ, USA: CRC press, 2005.

[13] A. El-Hoiydi and J.-D. Decotignie, "WiseMAC: An ultra low power MAC protocol for the downlink of infrastructure wireless sensor networks," in Proc. 9th Int. Symp. Computers and Communications, 2004.

[14] M. Buettner, G. V. Yee, E. Anderson, and R. Han, "X-MAC: A short preamble MAC protocol for duty-cycled wireless sensor networks," in Proc. 4th Int. Conf. Embedded Networked Sensor Systems, Boulder, CO, USA, 2006.

[15] Y. Sun, O. Gurewitz, and D. B. Johnson, "RI-MAC: A receiverinitiated asynchronous duty cycle MAC protocol for dynamic traffic loads in wireless sensor networks," in Proc. 6th ACM Conf. Embedded Network Sensor Systems, Raleigh, NC, USA, 2008.

[16] L. Tang, Y. Sun, O. Gurewitz, and D. Johnson, "PW-MAC: An energy-efficient predictive-wakeup MAC protocol for wireless sensor networks," in Proc. IEEE INFOCOM, Shanghai, China, 2011.

[17] P. Gupta and P. Kumar, "The capacity of wireless networks," IEEE Trans. Inf. Theory, vol. 46, no. 2, pp. 388-404, Mar. 2000.

[18] L.-L. Xie and P. Kumar, "A network information theory for wireless communication: Scaling laws and optimal operation," IEEE Trans. Inf. Theory, vol. 50, no. 5, pp. 748-767, May 2004.

[19] J. Andrews, S. Weber, M. Kountouris, and M. Haenggi, "Random access transport capacity," IEEE Trans. Wireless Commun., vol. 9, no. 6, pp. 2101-2111, Jun. 2010. 
[20] R. Bruno, M. Conti, and E. Gregori, "Optimal capacity of p-persistent CSMA protocols," IEEE Commun. Lett., vol. 7, no. 3, pp. 139-141, Mar. 2003.

[21] C.-K. Chau, M. Chen, and S.-C. Liew, "Capacity of large-scale CSMA wireless networks," IEEE/ACM Trans. Netw., vol. 19, no. 3, pp. 893-906, Jun. 2011.

[22] J.-B. Seo, H. Jin, and V. Leung, "Throughput upper-bound of slotted CSMA systems with unsaturated finite population," IEEE Trans. Commun., vol. 61, no. 6, pp. 2477-2487, Jun. 2013.

[23] S. C. Liew, C. H. Kai, H. C. Leung, and P. Wong, "Back-ofthe-envelope computation of throughput distributions in CSMA wireless networks," IEEE Trans. Mobile Comput., vol. 9, no. 9, pp. 1319-1331, Sept. 2010.

[24] R. MacKenzie and T. O'Farrell, "Throughput and delay analysis for p-persistent CSMA with heterogeneous traffic," IEEE Trans. Commun., vol. 58, no. 10, pp. 2881-2891, Oct. 2010.

[25] P. K. Wong, D. Yin, and T. Lee, "Analysis of non-persistent CSMA protocols with exponential backoff scheduling," IEEE Trans. Commun., vol. 59, no. 8, pp. 2206-2214, Aug. 2011.

[26] L. Jiang and J. Walrand, "A distributed CSMA algorithm for throughput and utility maximization in wireless networks," IEEE/ACM Trans. Netw., vol. 18, no. 3, pp. 960-972, Jun. 2010.

[27] S. Rajagopalan, D. Shah, and J. Shin, "Network adiabatic theorem: An efficient randomized protocol for contention resolution," in Proc. SIGMETRICS, New York, NY, USA, 2009, pp. 133-144.

[28] J. Ni, B. Tan, and R. Srikant, "Q-CSMA: Queue-length-based CSMA/CA algorithms for achieving maximum throughput and low delay in wireless networks," IEEE/ACM Trans. Netw., vol. 20, no. 3, pp. 825-836, Jun. 2012.

[29] R. Boorstyn, A. Kershenbaum, B. Maglaris, and V. Sahin, "Throughput analysis in multihop CSMA packet radio networks," IEEE Trans. Commun., vol. 35, no. 3, pp. 267-274, Mar. 1987.

[30] F. P. Kelly, "Stochastic models of computer communication systems," J. Roy. Statist. Soc. B, Methodol., vol. 47, no. 3, pp. 379-395, 1985.

[31] F. P. Kelly, "Loss networks," Ann. Appl. Probab., vol. 1, no. 3, pp. 319-378, 1991

[32] F. Viger and M. Latapy, "Efficient and simple generation of random simple connected graphs with prescribed degree sequence," in Computing and Combinatorics. Berlin, Germany: Springer, 2005

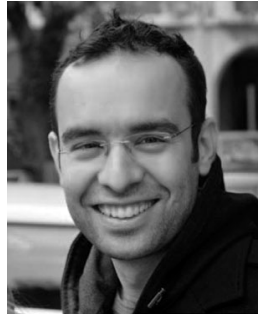

Mehmet Koseoglu received the B.Sc., M.Sc. and Ph.D. degrees from Bilkent University, Ankara, Turkey, all in Electrical and Electronics Engineering in 2004, 2007 and 2013, respectively. From 2004 to 2006, he was with software engineer for Aselsan Inc., Ankara, Turkey. During 2007-2013, he was a research and teaching assistant at the Electrical and Electronics Engineering Department of Bilkent University where he is currently a Post-Doctoral Researcher. His current research interests include on the performance analysis of wireless multiple access schemes.

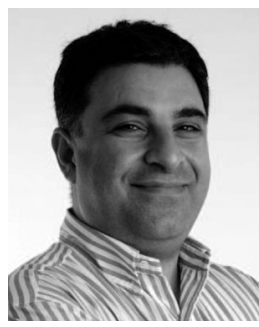

Ezhan Karasan received the B.S. degree from Middle East Technical University, Ankara, Turkey, the M.S. degree from Bilkent University, Ankara, Turkey, and the Ph.D. degree from Rutgers University, Piscataway, NJ, USA, all in electrical engineering, in 1987, 1990, and 1995, respectively. During 1995-1996, he was a PostDoctorate Researcher at Bell Labs, Holmdel, NJ, USA. From 1996 to 1998, he was a Senior Technical Staff Member in the Lightwave Networks Research Department at AT\&T LabsResearch, Red Bank, NJ, USA. He has been with the Department of Electrical and Electronics Engineering at Bilkent University since 1998 , where he is currently a full professor. He is a member of the Editorial Board of Optical Switching and Networking journal. He is the recipient of 2004 Young Scientist Award from Turkish Scientific and Technical Research Council (TUBITAK), 2005 Young Scientist Award from Mustafa Parlar Foundation and Career Grant from TUBITAK in 2004. He received a fellowship from NATO Science Scholarship Program for overseas studies in 1991-1994. He has participated in FP6IST Network of Excellence (NoE) e-Photon/ONe+ and FP7-IST NoE BONE projects. His current research interests are include in the application of optimization and performance analysis tools for the design, engineering and analysis of optical and wireless networks. He is a member of the IEEE.

$\triangleright$ For more information on this or any other computing topic, please visit our Digital Library at www.computer.org/publications/dlib. 